\title{
GENERALIZED WHITTAKER'S EQUATIONS FOR HOLONOMIC MECHANICAL SYSTEMS
}

\author{
MUNAWAR HUSSAIN \\ Department of Mathematics \\ Government College \\ Lahore, Pakistan \\ (Received June 23, 1977)
}

ABSTRACT. In this paper the classical theorem "a conservative holonomic dynamic system is invariantly connected with a certain differential form" 1s generalized to group variables. This general theorem is then used to reduce the order of a Hamiltonian system by the use of the integral of energy. Equations of motion of the reduced system so obtained are derived which are the so-called generalized Whittaker's equations. Finally an example is given as an application of the theory.

KEY WORDS AND PHRASES. Generalized Whittaker's equations, Holonomic Systems. AMS (MOS) SUBJECT CLASSIFICATION (1970). 70F20

1. INTRODUCTION

It is known [4] that canonical equations for a conservative holonomic system whose Hamiltonian is $H$ are obtained by forming the first Pfaff's system of differential equations of the differential form,

$$
p_{1} d q_{1}-H d t, \quad(1=1,2,-\cdots, n),
$$


where $p_{1}, p_{2},--, p_{n}$ are the generalized momenta corresponding to the generalized coordinates $q_{1}, q_{2}, \cdots, q_{n}$ of the system and summation over a repeated suffix 18 implied. Th1s result leads to the theorem "A dynamical system is invarlantly connected with the differential form $p_{1} d q_{1}$ - Hdt". Further this theorem has been used to reduce the order of the system by means of the integral of energy. Canonical equations of the reduced system so obtained are known as Whittaker's equations. In what follows in this section we state a few basic results from the theory of group variables in order to generalize the above mentioned results.

Consider a conservative holonomic system having $n$ degrees of freedom and whose position 18 spectfied by group variables $x_{1}, x_{2}, \cdots, x_{n}$. Let $n_{1}, n_{2}, \cdots, n_{n}$ be the parameters of real displacement and $x_{1}, x_{2},-\cdots, x_{n}$ be the corresponding displacement operators expressed by the relations

$$
x_{1}=\xi_{1} \frac{\partial}{\partial x_{j}}, \quad(1, j=1,2, \cdots, n)
$$

where $\xi_{1}$ are functions of $x_{1}, x_{2},--, x_{n}$, then for an arbitrary function $f\left(x_{1}, x_{2}, \cdots, x_{n}, t\right)$ the infinitesimal change df is expressed by

$$
d f=\left[x_{0}(f)+n_{1} x_{1}(f)\right] d t
$$

where $x_{0}=\frac{\partial}{\partial t}$. The $X^{\prime} s$ satisfy the relations

$$
\left(x_{0}, x_{1}\right)=0,\left(x_{1}, x_{j}\right)=c_{1 j k} x_{k} \quad(k=1,2, \ldots, n) .
$$

Putting $f=x_{f}$ in (2), we get

$$
\frac{d x_{j}}{d t}=\dot{x}_{j}=\cdot n_{1} \frac{j}{\xi_{i}}
$$

Strce the operators $x_{1}$ are independent therefore the matrix $\left\|\xi_{1}\right\|$ is nonsingular and consequently (4) yields

$$
n_{1}=A_{1 j} x_{j}
$$


Let $L$ be the Lagrangian of the system then the canonical equations of the system as obtained in $[1,2]$ are

$$
n_{1}=\frac{\partial H}{\partial y_{1}}, \frac{d y_{1}}{d t}=c_{j i k} n_{j} y_{k}-x_{1}(H) \text {, }
$$

where

$$
(1, j, k=1,2,---, n),
$$

$$
y_{1}=\frac{\partial L}{\partial n_{1}}
$$

and

$$
\mathrm{H}\left(\mathrm{x}_{1}, \mathrm{x}_{2}, \cdots, \mathrm{x}_{\mathrm{n}} ; \mathrm{y}_{1},-\cdots, \mathrm{y}_{\mathrm{n}}\right)=n_{1} \mathrm{y}_{1}-\mathrm{L}
$$

is the Hamiltonian of the system and is equal to the total energy of the system. 2. DERIVATION OF CANONICAL EQUATIONS FROM A CERTAIN DIFFERENTIAL FORM.

In order to establish the invariant relation between the system (6) and a certain differential form we prove the following theorem:

THEOREM. The system of equations (6) is equivalent to the first Pfaff's system of differential equations of the differential form $\left(n_{1} y_{2}-H\right) d t$.

PROOF. We put

$$
\theta_{d}=\left(n_{1} y_{1}-H\right) d t
$$

or using (5), we obtain

$$
\theta_{d}=y_{1} A_{1 j} d x_{j}-H d t
$$

therefore

$$
{ }_{\delta}=y_{1} A_{1 j} \delta x_{j}-H \delta t
$$

where $d$ and $\delta$ denote two independent variations in each of the variables $x_{1}$, $x_{2}, \cdots, x_{n}, y_{1}, \cdots, y_{n}, t$. The bilinear coreariant of (8) is given by

$\delta \theta_{d}-d \theta_{\delta}=\delta y_{1}\left[A_{1 j} d x_{j}-\frac{\partial H}{\partial y_{1}} d t\right]+\delta x_{k}\left[y_{1} \frac{\partial A_{1 j}}{\partial x_{k}} d x_{j}-\frac{\partial H}{\partial x_{k}} d t-d y_{1} A_{1 k}-y_{1} \frac{\partial A_{1 k}}{\partial x_{j}} d x_{j}\right]$

$+\delta t\left[d H-\frac{\partial H}{\partial t} d t\right]$ 
where we have used the relations

$$
\begin{aligned}
& d \delta x_{1}=\delta d x_{1}(1=1,2,-\cdots, n) \\
& d \delta t=\delta d t .
\end{aligned}
$$

Equating to zero the coefficlents of $\delta x_{1}, \delta x_{2}, \cdots, \delta x_{n}, \delta y_{1}, \cdots, \delta y_{n}, \delta t$ in $(10)$, we get the first Pfafe's system of equation in the form

$$
\begin{gathered}
A_{1 j} d x_{j}-\frac{\partial H}{\partial y_{1}} d t=0, \quad(1=1,2,---, n) \\
y_{1} \frac{\partial A_{1 j}}{\partial x_{k}} d x_{j}-\frac{\partial H}{\partial x_{k}} d t-d y_{1} A_{1 k}-y_{1} \frac{\partial A_{1 k}}{\partial x_{j}} d x_{j}=0 \\
d H=\frac{\partial H}{\partial t} d t, \quad(1=1,2,--, n) .
\end{gathered}
$$

By virtue of (5), the equations (11) assume the form

$$
\eta_{1}=\frac{\partial H}{\partial y_{1}}, \quad(1=1,2,-\cdots, n) \text {. }
$$

With the help of (4), the equations (12) become

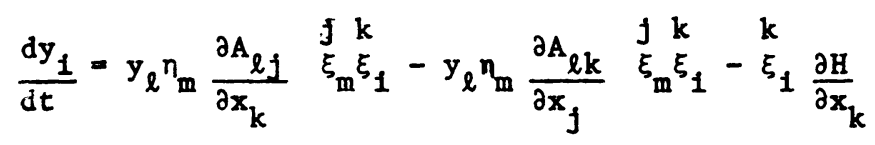

which, by means of the relations (1) and (3), finally takes the form

$$
\frac{d y_{1}}{d t}=n_{j} y_{k} c_{j i k}-x_{1}(H) \text {. }
$$

The relation (13) is a consequence of (14) and (15) and skew symmetric property of $C_{f i k}$ with respect to the first two indices. Since the equations (14) and (15) are identical with (6) the theorem is thus proved.

\section{GENERALIZED WHITTAKER'S EQUATIONS:}

Assume that $\mathrm{H}$ does not involve the time explicitly and

$$
\mathrm{H}+\mathbf{h}=0 \text {, }
$$

1s the Integral of energy of the system. Let the equation (16) be solved for the variable $y_{1}$ so that it is algebraically equivalent to

$$
k\left(x_{1}, \cdots, x_{n}, y_{2}, \cdots, y_{n}, t, h\right)+y_{1}=0 \text {. }
$$


The differential form assoclated with the system is

$$
\left(n_{1} y_{1}+n_{2} y_{2}+\cdots+n_{n} y_{n}+h\right) d t,
$$

where the variables $x_{1}, \ldots, x_{n}, y_{1}, \ldots, y_{n}, h$ are connected by (17); the differential form can therefore be written as

$$
\left(n_{2} y_{2}+n_{3} y_{3}+\cdots+n_{n} y_{n}+h\right) d t-n_{1} R d t
$$

where we can regard $\left(x_{1}, \ldots, x_{n}, y_{2}, \ldots, y_{n}, h, t\right)$ as the $2 n+1$ variables In the phase space. If we express (18) in the form

$$
n_{1} d t\left[h_{2}^{\prime} y_{2}+\cdots+n_{n}^{\prime} y_{n}+\frac{1}{n_{1}} h-k\right]
$$

and put

$$
n_{1} d t=d \tau
$$

then we take $\tau$ as the new time variable and $\frac{1}{n_{1}}, n_{1}^{\prime}=1, n_{2}^{\prime}=\frac{n_{2}}{n_{1}}, \ldots, n_{n}^{\prime}=\frac{n_{n}}{n_{1}}$

as the parameters of real displacement, the corresponding displacement operators and new momenta are respectively $x_{0}, x_{1}, \ldots, x_{n}$ and $h, y_{1}, \ldots, y_{n}$ Using the result of section (2), the differential equation corresponding to the form (19) are

$$
\begin{aligned}
& \eta_{p}^{\prime}=\frac{\partial K}{\partial y_{p}}, \frac{d y_{p}}{d \tau}=\eta_{j}^{\prime} y_{k} c_{j p k}-x_{p}(K), \quad(p=2,3,-\cdots, n) \\
& \frac{d t}{d \tau}=\frac{\partial K}{\partial h}, \frac{d h}{d \tau}=0 .
\end{aligned}
$$

The last pair of equations can be separated from the rest of the system since the first $(2 n-2)$ equations do not involve $t$ and $h$ is a constant. The equations (20) can be further simplified to take the form

$$
\begin{gathered}
n_{p}^{\prime}=\frac{\partial K}{\partial y_{p}}, \frac{d y_{p}}{d \tau}=-k\left[c_{1 p 1}+\eta_{r}^{\prime} c_{r p 1}\right]+y_{r} c_{1 p r}+n_{r}^{\prime} y_{q} c_{r p q}-x_{p}(K) \\
(p, q, r=2,3, \ldots, n) .
\end{gathered}
$$


The original differential equations can therefore be replaced by the reduced system (21) which has only n-1 degrees of freedom. The equations (21) are the desired Whittaker's equations.

\section{AN EXAMPLE}

Consider a rigid body which is moving about one of its fixed points 0 under the action of gravity. We introduce a fixed frame of reference Oxyz such that $0 z$ is vertically upwards and a moving frame $0 x^{\prime} y^{\prime} z$ ' which coincides with the principal axes of inertia of the body at 0 . Let us choose the Eulerian angles $\theta, \phi, \psi(\theta$ is the angle of nutation, $\phi$ the angle of precession and $\psi$ the angle of proper rotation) as the group varlables which specify the position of the body at time $t$. Obviously the dynamical system under consideration is a conservative one and it has three degrees of freedom. Choosing the parameters of real displacement as the components of angular velocity along the moving axes, we have the relations

$$
\begin{aligned}
& n_{1}=\dot{\theta} \cos \psi+\dot{\sin \theta \sin \psi,} \\
& n_{2}=-\dot{\theta} \operatorname{Sin} \psi+\dot{\phi} \sin \theta \cos \psi, \\
& n_{3}=\dot{\psi}+\dot{\phi} \cos \theta
\end{aligned}
$$

Consequently the displacement operators $x_{1}, x_{2}, x_{3}$ are given by

$$
\begin{aligned}
& \mathrm{x}_{1}=\operatorname{Cos} \psi \frac{\partial}{\partial \theta}+\operatorname{cosec} \theta \sin \psi \frac{\partial}{\partial \phi}-\cot \theta \sin \psi \frac{\partial}{\partial \psi} \\
& x_{2}=-\sin \psi \frac{\partial}{\partial \theta}+\operatorname{cosec} \theta \operatorname{Cos} \psi \frac{\partial}{\partial \phi}-\cot \theta \operatorname{Cos} \psi \frac{\partial}{\partial \psi} \\
& x_{3}=\frac{\partial}{\partial \psi}
\end{aligned}
$$

which satisfy the commutation relations

$$
\begin{aligned}
& \left(x_{1}, x_{2}\right)=x_{1} x_{2}-x_{2} x_{1}=x_{3} \\
& \left(x_{2}, x_{3}\right)=x_{2} x_{3}-x_{3} x_{2}=x_{1} \\
& \left(x_{3}, x_{1}\right)=x_{3} x_{1}-x_{1} x_{3}=x_{2}
\end{aligned}
$$


The non-vanishing C's are therefore expressed by the relations

$$
\left.\begin{array}{l}
c_{123}=-c_{213}=1, \\
c_{231}=-c_{321}=1, \\
c_{312}=-c_{132}=1 .
\end{array}\right]
$$

Let $T$ and $U$ denote the kinetic and potential energies of the system respectively, then

$$
\left.\begin{array}{l}
T=\frac{1}{2}\left(A n_{1}^{2}+B n_{2}^{2}+C n_{3}^{2}\right), \\
U=-M g(\bar{x} \sin \theta \sin \psi+\bar{y} \sin \theta \operatorname{Cos} \psi+\bar{z} \operatorname{Cos} \theta
\end{array}\right]
$$

where $A, B, C$ are the principal moments of inertia at $0 ; \bar{x}, \bar{y}, \bar{z}$ are the coordinates of the centre of gravity of the body with respect to the moving axis and $M$ is the mass of the body. Using (25), we have the Lagranglan $L$ and momenta $y_{1}, y_{2}, y_{3}$ expressed by the relations:

$$
\begin{gathered}
L=T-U=\frac{1}{2}\left(A n_{1}^{2}+B n_{2}^{2}+C \eta_{3}^{2}\right)+M g(\bar{x} \sin \theta \sin \psi+\bar{y} \sin \theta \operatorname{Cos} \psi+\bar{z} \operatorname{Cos} \theta), \\
y_{1}=A \eta_{1}, y_{2}=B \eta_{2}, y_{3}=C n_{3} .
\end{gathered}
$$

In view of (26) the Hamiltonian $\mathrm{H}$ is given by $\mathrm{H}=\frac{1}{2}\left(\frac{\mathrm{y}_{1}^{2}}{\mathrm{~A}}+\frac{\mathrm{y}_{2}^{2}}{\mathrm{~B}}+\frac{\mathrm{y}_{3}^{2}}{\mathrm{C}}\right)-\mathrm{Mg}(\overline{\mathrm{x}} \sin \theta \operatorname{Sin} \psi+\overline{\mathrm{y}} \sin \theta \operatorname{Cos} \psi+\bar{z} \operatorname{Cos} \theta)$ Using (6), (22), (24), (26), and (27), canonical equations of the system are

$$
\begin{aligned}
& n_{1}=\frac{y_{1}}{A}, n_{2}=\frac{y_{2}}{B}, n_{3}=\frac{y_{3}}{C} \\
& \frac{d y_{1}}{d t}=\frac{B-C}{B C} y_{2} y_{3}+M g(\bar{y} \operatorname{Cos} \theta-\bar{z} \sin \theta \cos \psi), \\
& \frac{d y_{2}}{d t}=\frac{C-A}{C A} y_{3} y_{1}+M g(-\bar{x} \cos \theta+\bar{z} \sin \theta \sin \psi) \\
& \frac{d y_{3}}{d t}=\frac{A-B}{A B} y_{1} y_{2}+M g \sin \theta(\bar{x} \operatorname{Cos} \psi-\bar{y} \sin \psi) .
\end{aligned}
$$


Now the relation (16) gives

$$
\frac{y_{1}^{2}}{A}+\frac{y_{2}^{2}}{B}+\frac{y_{3}^{2}}{C}-2 M g(\bar{x} \sin \theta \sin \psi+\bar{y} \sin \theta \operatorname{Cos} \psi+\bar{z} \operatorname{Cos} \theta)+2 h=0,
$$

and consequently

$$
y_{1}=A\left[2 M_{g}(\bar{x} \sin \theta \sin \psi+\bar{y} \sin \theta \operatorname{Cos} \psi+\bar{z} \operatorname{Cos} \theta)-\frac{y_{2}^{2}}{B}-\frac{y_{3}^{2}}{C}-2 h\right]^{\frac{1}{2}}
$$

Comparing this relation with (17), we get

$$
K=-\operatorname{A}\left[2 \mathrm{Mg}(\bar{x} \sin \theta \sin \psi+\bar{y} \sin \theta \cos \psi+\bar{z} \operatorname{Cos} \theta)-\frac{y_{2}^{2}}{B}-\frac{y_{3}^{2}}{C}-2 h\right]^{\frac{1}{2}} .
$$

Therefore by the application of (21) the canonical equations of the system reduce to

$$
\left.\begin{array}{l}
n_{2}^{\prime}=\frac{\partial K}{\partial y_{2}}, \quad n_{3}^{\prime}=\frac{\partial K}{\partial y_{3}}, \\
\frac{d y_{2}}{d \tau}=y_{3}-n_{3}^{\prime} y_{1}-x_{2}(K), \\
\frac{d y_{3}}{d \tau}=-y_{2}+n_{2}^{\prime} y_{1}-x_{3}(K) .
\end{array}\right]
$$

Now

$$
\begin{aligned}
& \frac{\partial K}{\partial h}=-\frac{A}{R}, \frac{\partial R}{\partial y_{2}}=-\frac{A}{B R} y_{2}, \frac{\partial R}{\partial y_{3}}=-\frac{A}{C K} y_{3}, \\
& \frac{d y_{2}}{d \tau}=\frac{d y_{2}}{d t} \frac{\partial R}{\partial h}=-\frac{A}{R} \frac{d y_{2}}{d t} \\
& \frac{d y_{3}}{d t}=\frac{d y_{3}}{d t} \frac{\partial K}{\partial h}=-\frac{A}{K} \frac{d y_{3}}{d t} \\
& x_{2}(K)=\frac{A}{\bar{K}} \cdot M g(-\bar{x} \cos \theta+\bar{z} \sin \theta \sin \psi), \\
& x_{3}(K)=\frac{A}{k} \cdot M g(\bar{x} \cos \psi-\bar{y} \sin \psi) \sin \theta \text {. }
\end{aligned}
$$


Therefore equation (29) assume the form

$$
\begin{aligned}
& \eta_{2}^{\prime}=-\frac{A}{B K} y_{2}, \quad \eta_{3}^{\prime}=-\frac{A}{C K} y_{3} \\
& \frac{d y_{2}}{d t}=\frac{K(A-C)}{C A} y_{3}+M g(-\bar{x} \cos \theta+\bar{z} \sin \theta \sin \psi), \\
& \frac{d y_{3}}{d t}=\frac{K(B-A)}{A B} y_{2}+M g \sin \theta(\bar{x} \cos \psi-\bar{y} \sin \psi) .
\end{aligned}
$$

These are the Whittaker's equations for the system under consideration.

\section{REFERENCES}

1. Cetaev, N. G. On the Equations of Poincare, Prikl. Mat. Meh. (1941) 253-262.

2. Hussain, M. Hamilton-Jacobi Theorem in Group Variables, Journal of Applied Mathematics and Physics (ZAMP), Vol. 27, (1976) 285-287.

3. Poincare, H. On a New Form of the Equations of Mechanics, C. R. Acad. Sc1. 132 (1901) 369-371.

4. Whittaker, E. T. Analytical Dynamics of Particles and Rigid Bodies, Cambridge University Press, 1961. 


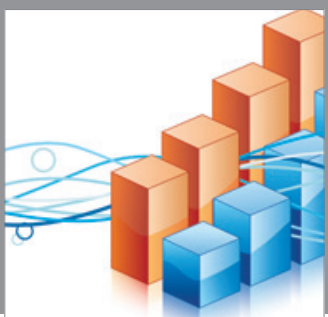

Advances in

Operations Research



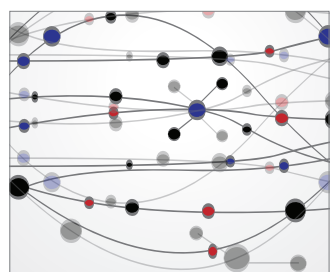

\section{The Scientific} World Journal
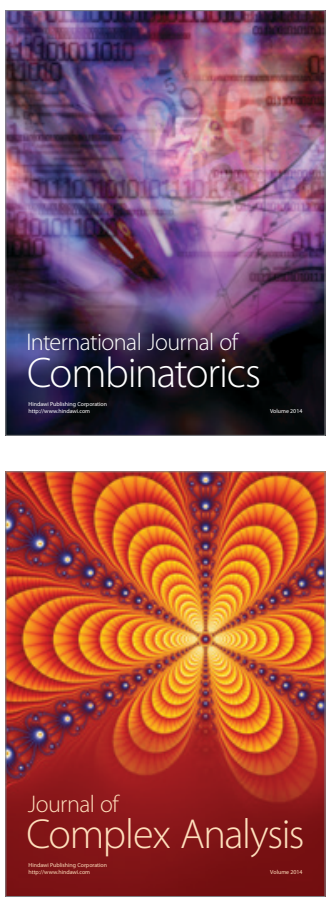

International Journal of

Mathematics and

Mathematical

Sciences
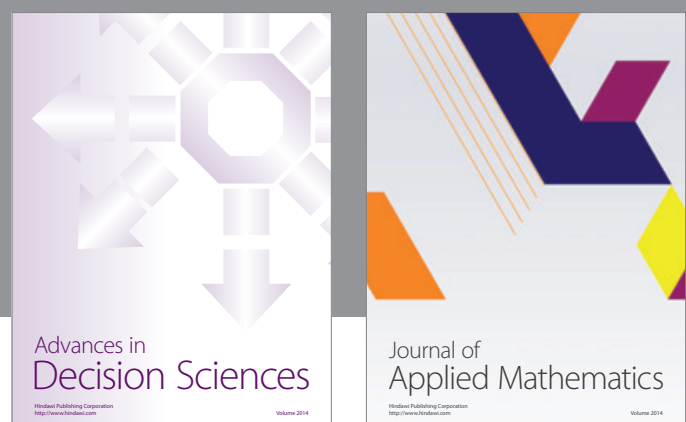

Journal of

Applied Mathematics
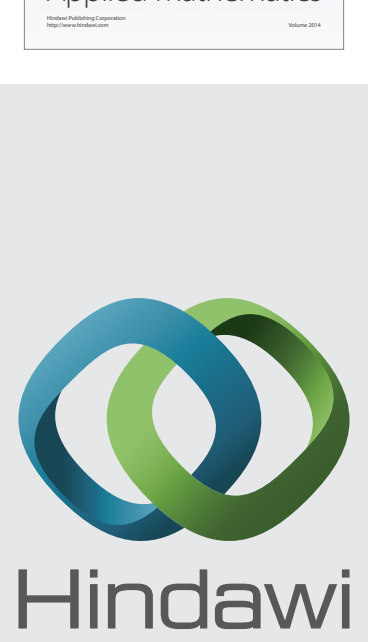

Submit your manuscripts at http://www.hindawi.com
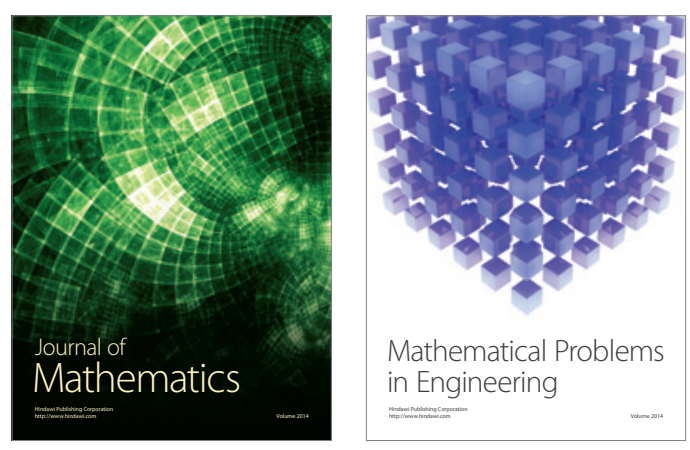

Mathematical Problems in Engineering


Journal of

Function Spaces
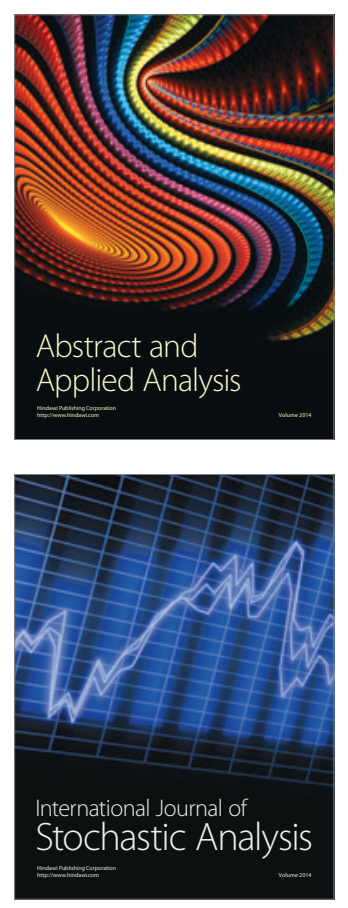

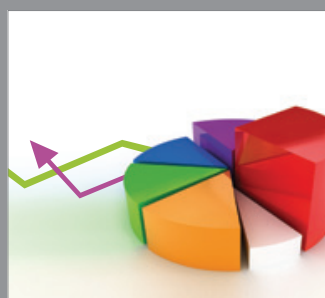

ournal of

Probability and Statistics

Promensencen
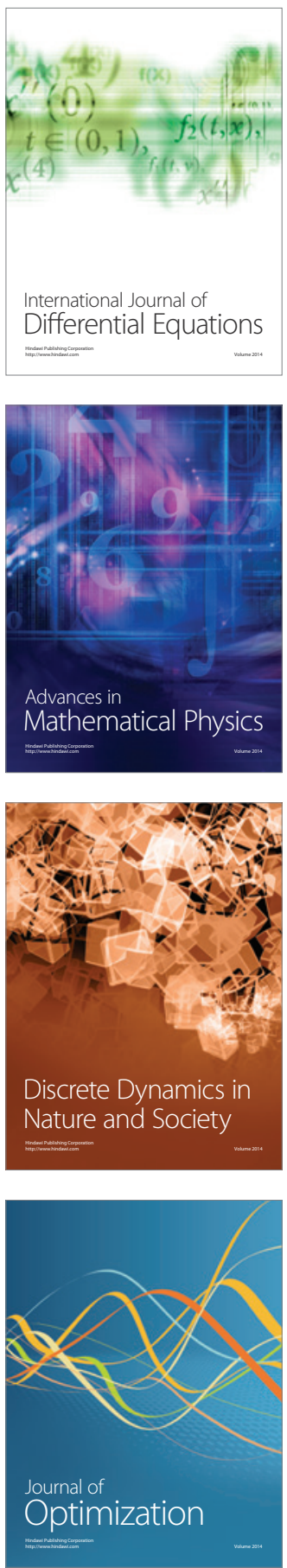